\title{
Wirkung des Coriariatoxins und Coriatoxins, der giftigen Bestandteile der Frucht der Coriaria japonica, A. Gray auf den Frosch.
}

\author{
Von \\ Sadao Miyachi. \\ (宮 地貞 雄) \\ (Aus dem Pharmakologischen Institut der Tohoku Reichs- \\ universität zu Sendai. Direktor: Prof. Dr. S. Yagi.)
}

Früher hatte ich' ${ }^{1}$ bereits mitgeteilt, dass die Frucht der Coriaria japonica, A. Gray verschiedene giftige Bestandteile enthält, deren Menge je nach der Reife der Frucht zu- und abnimmt, ferner dass die wesentlichen Bestandteile während der Erntezeit der Frucht verschieden sind und dass aus einem Gemisch dieser giftigen Bestandteile 2 Arten von Bitterstoffen, das Coriariatoxin und Coriatoxin, rein isoliert werden konnten. Von diesen ist das erstere in den Eigenschaften dem von Kariyone ${ }^{2) *}$ aus der Frucht derselben Pflanze isolierten Tutin analog, aber im Schmelzpunkt verschieden, indem die von diesem Forscher gewonnene Substanz bei $213^{\circ} \mathrm{C}$, während die von mir erhaltene, wie oft ich sie auch umkrystallisierte, bei $209^{\circ} \mathrm{C}$ schmilzt, sodass man die beiden nicht als mit einander identisch bezeichnen kann. Das Coriatoxin hat einen Schmelzpunkt von $275^{\circ} \mathrm{C}$; diese Angabe ist in der bisherigen Mitteilung nicht enthalten.

Da diese beiden Substanzen auf Frösche krampfauslösend wirken; anscheinend ganz wie das Tutin oder Coriamyrtin, die aus der Pflanze gleicher Gattung isoliert werden, so müssten die ersteren auch in ihren sonstigen Wirkungen den letzteren ganz oder annähernd gleich sein, wofür aber noch kein sicherer Anhalt vorliegt. Dies hat mich veranlasst, zuerst die Wirkungen dieser Substanzen an Fröschen zu beobachten und sie mit denen des Tutins und Coriamyrtins zu verglei-

* Zur Anstellung der vergleichenden Versuche hat Herr T, Karig o ne, an der hygienischen Untersuchungsstation des inneren Ministeriums, mich mit wertvollem Material beschenkt, wofür ich ihm auch an dieser Stelle meinen herzlichsten Dank ausspreche. 
chen. Da die Versuche ergaben, dass das Coriariatoxin und Coriatoxin qualitativ gleiche, aber an Stärke verschiedene Wirkungen entfalten, so will ich zuerst über die erstere Substanz berichten und dann die Punkte hinzufügen, in welchen sich die letztere verschieden erweist.

\section{Wirkung des Coriariatoxins.}

Das Coriariatoxin ruft bei zwei Froscharten, die Rana nigromaculata und die Rana japonica, zuerst spontane, dann Reflexkrämpfe, zugleich Beschleunigung der Atembewegungen, Steigerung der Hautsekretion und Pupillenerweiterung hervor. Nachher erzeugt das Gift Abschwächung der willkürlichen Reflex- und Atembewegungen bis zu ihrem Verschwinden, Herabsetzung der Hautabsonderung, Pupillenverengerung, und schliesslich Tod durch Herzstillstand.

Die minimale letale Dose des Giftes ist je nach den Jahreszeiten, in denen der Versuch angestellt wird, sowie den Froscharten mehr oder minder verschieden, und beträgt bei der Rana nigromaculata bei Darreichung in den Bauchlymphsack pro $100 \mathrm{~g}$ Körpergewicht im Februar 0,6, im Mai 0,15 und im September 0,23 mg. Die kleinste krampferzeugende Dose entspricht ca. einem Zehntel der obigen Dosen. Auch der Verlauf der Vergiftung bei Anwendung der minimalen tödlichen Dose ist je nach den Jahreszeiten verschieden, im Mai und September ist er kurz und beläuft sich auf 17 Stunden, im Februar dagegen ist er länger und beträgt 24 Stunden. Im allgemeinen ist die Rana japonica empfindlicher, und der Vergiftungsverlauf ist kürzer als bei der Rana nigromaculata.

Um den Verlauf der obigen Symptome und die Beziehung derselben zu einander zu veranschaulichen, wird im folgenden das Protokoll eines Versuches an einer Rana nigromaculata wiedergeben, die im Februar die tödliche Dose des betreffenden Giftes bekommen hat. Dieser Versuch ist deshalb herausgegriffen worden, weil die Vergiftung im Winter, wie oben erwähnt, langsam verläuft und die Symptome in richtiger Reihenfolge erkennbar sind.

Rana nigromaculata, $\hat{o}, 15 \mathrm{~g}$.

$7 \mathrm{~h} 30^{\prime}$ a.m. den 12 . Febr.: Injektion von $0,5 \mathrm{ccm} 0,002 \%$ iger Coriariatoxinlösung in den Bauchlymphsack; unmittelbar danach springt das Tier umher, aber nicht dauernd.

37' Ruhig, Atembewegungen sind bedeutend rascher, Brust und Bauch sind etwas aufgetrieben.

45' Spontane Bewegungen werden kaum ausgeführt, die Atemfrequenz nimmt ab, die Atembewegungen sind unregelmässig.

55' Das Tier liegt flach mit dem Bauch auf dem Boden, Atembewegungen 
sind selten. Es beugt ab und zu den Kopf nach dem Rücken, abduziert die Hinterextermitäten und verschiebt sich mit gespreizten Zehen.

8h15' do.

$30^{\prime}$ Brust und Bauch sind stark aufgebläht, zuweilen zeigen sich unter dem Kiefer und an der Bauchseite feine fibrilläre Zuckungen. Dann und wann wiederholen sich die oben beschriebenen Bewegungen, sie werden mit der Zeit stärker, der Kopf wird stark dorsal gebeugt, die Vorder- und Hinterextremitäten werden nach beiden Seiten und die Hinterextremitäten gleichzeitig nach oben gespreizt, sie zeigen klonische Krämpfe, und das Tier macht infolgedessen den Eindruck, als ob es den ganzen Körper mit dem Bauch stiitzte und sich drehte. Nach Abklingen der Krämpfe werden mehrmals tiefe Atmungen ausgeführt.

35' Die Anfälle der klonischen Krämpfe werden häufiger. Die Hant wird feuchter, die Pupillen sind erweitert.

$43^{\prime}$ Sobald das Tier unruhig wird, springt es einige Male in die Höhe, zeigt einen lauten knarrenden Schrei hervorbringend tonische Krämpfe, dabei werden Brust und Bauch eng und das Tier beugt den Kopf stark nach oben, ebenso krümmt es den Rücken konkav nach oben, streckt die Vorderextremitäten nach beiden Seiten und die Hinterextremitäten, die Zehen spreizend nach hinten und oben. Die Atmung hört ganz auf, die Pupillen werden etwas verengt. Diese Krämpfe gehen nachher in klonische über, die Dauer derselben betägt ca. 1 Minute.

Beim Aufhören der Krämpfe erfolgen mehrmals tiefe Atmungen, zuweilen wird das Maul weit geöffnet, die Pupillen werden enger, die Haut wird bedeutend feuchter, die Berührungsstelle des Oberschenkels mit den Bauchseiten bedeckt sich mit Schaum.

$45^{\prime}$ Anfalle von tonischen und klonischen Krämpfen von ca. $1 \frac{1}{2}$ Minuten Dauer. Seitdem wiederholen sich gleichartige Krampfanfälle.

9h-Die Krampfanfälle werden immer kürzer und schwächer, und ihre Intervalle länger. In den Intervallen bleiben die willkürlichen und die Reflexbewegungen aus.

$30^{\prime}$ Die Krampfanfälle bleiben aus, nur selten treten an den Hinterextremitäten schwache fibrilläre Zuckungen auf.

$10^{\mathrm{h}}$ - Der ganze Körper erschlafft und das Tier liegt platt mit dem Bauch auf dem Boden, es macht keine Bewegungen, reagiert aber auf mechanische Reize, indem es den Kopf ventral beugt und eine schwache Bewegung macht, als ob es die Hinterextremitäten strecken wollte. Die Atembewegungen fehlen ganz uud die Pupillen sind stark verengt.

$30^{\prime}$ do. Die Haut wird trocken, an der Brust erkennt man reglemässigen Herzschlag.

$12^{\mathrm{h}}-$ do.

$2^{\mathrm{h}}$ p.m. do.

$4 \mathrm{~h}$ do.

6h- Das Tier liegt ganz erschlafft auf dem Bauch, auf mechanische Reize reagiert es mit ziemlich starken Bewegungen des Kopfes und der Extremitäten.

$8^{\text {h }}$ - Das Tier liegt mit dem Bauch platt auf dem Boden, die Haut wird wieder feucht, die Pupillen werden wieder erweitert, auf mechanische Reize erfolgen immer krampfartige Bewegungen, und zuweilen sogar heftige tonische Krämpfe in opisthotonischer Haltung. 
$8 \mathrm{~h} 30^{\prime}$ do.

9h Auf mechanische Reize reagiert das Tiere nur mit schwachen Bewegungen der Extremitäten.

$10^{\mathrm{h}}$ Das Tier liegt platt auf dem Bauch, die Haut wird wieder trocken und die Pupillen werden verengt, auf mechanische Reize reagiert es gar nicht, aber an der Brustwand kann man den Herszchlag, wenn auch schwach, dauernd konstatieren. Mikroskopisch erkennt man in der Schwimmhaut den Blutkreislauf.

7h- a.m. den 13. II. Das Herz steht still.

Im folgenden werden die geschilderten einzelnen Symptome einer genaueren Beobachtung unterzogen, um ihre Entstehungsweise zu erklären.

\section{Die Krämpfe.}

Wie das Versuchsprotokoll deutlich zeigt, können die durch das Coriariatoxin herbeigeführten Krämpfe in solche eingeteilt werden, die sich im Anfangsstadium anfallsweise einstellen und solche, die im Späterstadium nach dem völligen Schwund der ersten Krämpfe von neuem einsetzen. Die ersteren, welche durch äussere Reize verstärkt, aber auch ohne dieselben hervorgeruhen werden, sind von klonischem Charakter, und bestehen darin, dass das Tier den Rumpf konkav nach oben krümmt, die Extremitäten nach beiden Seiten streckt, und die Zehen bis zum Äussersten spreizt. Bei den starken Anfällen treibt das Tier die in der Brust angesammelte Luft auf einmal mit lautem, knarrendem Schrei aus, und die Krämpfe werden dann tonische, in gleicher Weise wie die durch Pikrotoxin verursachten. Die im Spätstadium auftretenden Krämpfe zeigen sich selten ohne bemerkbare äussere Reize, sondern meistens durch dieselben. Dabei wird der Rumpf konkav nach oben gekrümmt, die Hinterextremitäten gerado nach hinten gestreckt, die Zehen jedoch nicht so auffallend gespreizt wie bei den klonischen Krämpfen. Es zeigen sich die tetanischen Streckkrämpfe gerade wie bei der Strychninvergiftung.

Zwecks Untersuchung der Entstehungsweise dieser Krämpfe stellte ich folgendes Experiment an: Ich öffnete an einem Frosch den Schädel, legte das Gehirn bloss und bestrich es an der Oberfläche mit $0,1 \%$ iger Coriariatoxinlösung; dann stellten sich nach etwa zehn Minuten Krämpfe ein, die denen durch das Pikrotoxin ähnlich sind. Wird aber das Tier intakt gelassen, so erholt es sich von den Krämpfen und fällt niemals den strychninähnlichen Krämpfen anheim. Beim Bestreichen des Lobus opticus des freigelegten Gehirns bloss am vorderen Teile der Oberfläche mit der obigen Lösung zeigen sich keine Krämpfe. Gibt man aber einem Tiere mit unmittelbar hinter dem 
Lobus opticus quer durchschittenen Gehirn in den Bauchlymphsack die tödliche Dose dieses Giftes, so treten wie bei normalen Tieren zuerst pikrotoxinähnliche und später strychninähnliche Krämpfe in Erschei nung. Wenn ferner das blossgelegte verlägerte Mark an der Oberfläche mit 0,1\% iger Giftlösung bestrichen wird, setzen die ersteren Krämpfe ein, nicht aber die letzteren. Gibt man einem Tiere, dem in der Nähe des Rückenmarks die Medulla oblongata durchschnitten wurde, die tödliche Dose des betreffenden Giftes subkutan, so treten, ohne dass die pikrotoxinähnlichen Krämpfe entstehen, im Spätstadium strychninähnliche auf, die durch Zerstörung des Rückenmarks sofort zurückgehen.

Nach diesen Versuchsergebnissen entstehen die durch das Coriariatoxin im Anfangsstadium einsetzenden pikrotoxinähnlichen Krämpfe durch Erregung des verlängerten Markes und die anderen Hirnteile sowie das Rückenmark nehmen an ihnen nicht teil. Die Steigerung der Reflexbewegungen oder die tetanischen Krämpfe, die im Spätstadium auftreten, rühren von der erhöhten Reflexerregbarkeit des Rückenmarks her. Es ist aber nicht klar, welcher Prozess dafür verantwortlich ist, dass die einmal abgeschwächten Reflexbewegungen später gesteigert und so tonische Krämpfe ausgelöst werden. Es liegt jedoch nahe anzunehmen, dass das Coriariatoxin, wenn es auch die Erregbarkeit eines Teiles der den Rückenmarksreflexbogen bildenden Bahn herabsetzt, vor der totalen Lähmung desselben die Erregbarkeit des anderen Teiles erhöht, infolgedessen zuerst die Abschwächung der Reflexbewegungen und nachher die Steigerung derselben bewirkt.

Daraus geht hervor, dass die Krämpfe im Anfangsstadium nicht nur scheinbare sind, sondern auch in ihrer Entstehungsweise solchen durch das Pikrotoxin, Coriamyrtin und Tutin äusserst ähneln. Indessen beweist die Tatsache, dass das Pikrotoxin auch bei einem Tiere noch klonische Krämfe hervorruft, dem zwischen dem verlängerten und Rückenmark das Gehirn durchschnitten wurde (Gottlieb), ${ }^{3)}$ dass das Coriariatoxin näher mit dem Coriamyrtin und dem Tutin verwandt ist, als mit dem Pikrotoxin, da bei ersteren die klonischen Krämpfe nach Durchschneidung des Rückenmarks gänzlich ausbleiben (Marshall).4) Auch wirkt das Gift auf den Riickenmarksreflex steigernd, und zwar unabhänig davon, ob die Verbindung zwischen der Medulla oblongata und dem Rückenmark durchschnitten istodernicht, während das Pikrotoxin nur bei einem Tiere mit durchschnittenem Rückenmark diese Wirkung zu entfalten scheint (Guinard u. Dumarest), ${ }^{5}$ was man auch als die Eigenschaft bezeichnen kann, durch welche Coriariatoxin sich vom Pikrotoxin unterscheidet. Ob das Coriamyrtin und Tutin solche Eigenschaften besitzen oder nicht, ist vorläufig unbe- 
kanit eind diesbezügliche Mitteilung konnte nicht aufgefunden werden.

.
$1\}$
2. Die motorische Lähmung.

Wiébengesagt, kommen bei der Anwendung des Coriariatoxins in grossex D/sen nach Wiederholung von eigentümlichen Krämpfen sponterne to Reflexbewegungen vollkommen zum Schwund, trotzdem das 14erz egelmässig fortschlägt, wenn auch langsam und schwach, und 10 Blutströmung in der Schwimmhaut beobachtet werden kann,

tass man annehmen muss, dass die genannte motorische Störung offenbar nicht durch eine Kreislaufstörung bedingt ist, sondern durch die Giftwirkung. Wenn dabei der Gastrocnemiums und Ischiadicus blossgelegt und auf die Erregbarkeit für elektrischen Reize geprüft werden, so ist dieselbe nachweislich von einer solchen bei normalen Tieren nicht sehr verscheiden. Die elektrische Reizung der Wirbel säule ruft aber keine Muskelzuckung der Hinterextremitäten hervor. Es unterliegt also keinem Zweisel, dass die spontanen und Reflexbewegungen durch Rückenmarkslähmung zum Rückgang gebracht werden, wenn auch unklar ist, ob sich am Verschwinden der Bewegungen noch höher liegende Teile des Zentralnervensystems ebenfalls beteiliegen oder nicht.

\section{Die Atembewegungen.}

Die Atembewegungen beginnen nach der Giftanwendung bereits zuzunehmen bevor die anderen Veränderungen deutlich auftreten, und werden manchmal zu der Zeit, wo der Beruhigungszustand einsetzt, bedeutend vermehrt, bis ihre Frequenz doppelt so gross wird wie in der Norm.

Diese Zunahme ist eine vorübergehende, ihr folgt eine Abnahme und eine unregelmässige Atmung sowie Atmungsstillstand vor dem Eintritt der pikrotoxinähnlichen Krämpfe. Nach Auftreten der Krampfanfälle erfolgen in den Zwischenzeiten im Anschluss an diese Anfälle einige tiefe A tembewegungen. Diese Atembewegungen stellen sich aber nicht ein, wenn die pikrotoxinartigen Krampanfälle nicht mehr eintreten. Wenn aber die Krampfanfälle durch Rückenmarksreflexsteigerung wieder einsetzen, so erfolgen wiederum umittelbar nach dem Abklingen der Krämpfe zwischen den Anfällen einige Atembewegungen, welche jedoch nach dem Verschwinden dieser Krampfanfälle nicht mehr auftreten.

Daraus folgt, dass das Coriariatoxin durch Erregung des Atemzentrums die Atembewegungen zunächst vermehrt und verstärkt, die- 
selben dann langsam durch Lähmung desselben abschwächt und verschwinden lässt. Dass unmittelbar nach den Krämpfen Respirationsbewegungen erfolgen, ist wohl eine Folge davon, dass das nicht vollkommen gelähmte Atemzentrum durch die mit den Krämpfen einhergehende Kreislaufstörung oder durch veränderte Blutzusammensetzung eine Erregung erfährt.

\section{Das Herz.}

Auch nach vollkommener motorischer Lähmung durch das Coriariatoxin pulsiert das Herz noch weiter, wenn auch langsam und schwach und steht erst nach sehr langer Zeit still, wie schon oben angegeben wurde. Um zu untersuchen, ob sich seine Schläge im Verlaufe der Vergiftung verändern, wurde einem Frosch mit blossgelegtem Herzen in den Oberschenkellymphsack die letale Dose dieses Giftes injiziert und die dadurch erfolgende Beeinflussung der Herzschläge beobachtet. Dabei ergab sich, dass sich die Pulsationen kaum verändern, bis post injectionem deutliche Krampfanfälle einsetzen. Aber während der letzteren werden die Herzschläge langsam und unregelmässig und das Herz, welches reichlich mit Blut gefüllt ist, gerät in den Zustand, in dem es keine vollkommenen Kontraktionen ausführen kann. Solche Veränderungen wiederholen sich zusammen mit dem Auftreten der Krampfanfälle und gehen mit der Abschwächung derselben zurück, aber ungefähr zu der Zeit, wo dieselben verschwinden, werden die Pulsationen sehr langsam. Beim Wiedereinsetzen der spinalen Reflexe stellen sich bei jedem Anfalle wieder analoge Veränderungen ein und gehen mit dem Verschwinden desselben zurück. Danach werden die Herzschläge allmählich langsamer und schwächer, bis schliesslich der Stillstand erfolgt. Diese Erscheinungen werden auch bei den atropinisierten Tieren beobachtet, sodass man annehmen muss, dass ihre Ursache hauptsächlich in einem anderen Umstande als dem der Vaguserregungen zu suchen ist, wenn auch eine etwaige Beteiligung der Vagusreizung an ihrem Auftreten nicht ausgeschlossen werden kann. Beim curarisierten Tiere werden die Herzschläge von Anfang an langsamer und irregulär, bis sie schliesslich vollkommen aufhören, das Herz gerät aber nicht in den Zustand der erschwerten Blutaustreibung. Also scheint die während der Krampfanfälle entstehende Unmöglichkeit der Austreibung daher zu rühren, dass durch die Kontraktionen der Skelettmuskeln und auch wohl durch die Erregung des Gefässzentrums die Gefässe verengt und dadurch eine übermässig grosse Resistenz gegen das Herz erzeugt wird. Dafür dürfte auch die Tatsache sprechen, dass beim Einwirkenlassen einer Coriariatoxinlösung $(1: 200)$ auf das isolierte Froschherz inner- 
halb einer Stunde die Herzschläge nur relativ wenig abgeschwächt werden, und nicht die Veränderungen der oben geschilderten Art erfahren. Das Coriariatoxin hat also nicht die Eigenschaft den Herzvagus nachweisbar zu erregen, im Gegensatz zum Pikrotoxin, von welchem angegeben wird, dass es durch Vagusreizuug einen vorübergehenden Herzstillstand hervorruft (Röber). ${ }^{6}$

\section{Die Pupillen.}

Die Pupillenweite wurde in der Dunkelkammer mit konstanter Lichtquelle untersucht, da ihre Beobachtung im Tageslicht während der allgemeinen Vergiftungserscheinungen unsicher war. Dabei ergab sich, dass die Pupillen im allgemeinen einige Zeit post injectionem sich zu erweitern beginnen, danach sich langsam immer mehr dilatieren, um kurz vor dem Stadium der motorischen Lähmung die maximale Weite zu erreichen, sich aber in diesem Stadium etwas verengen, im späteren Krampfstadium wieder erweitern, und beim Verschwinden der Krämpfe sich aufs neue verengen, um im Endstadium bei weitem enger zu werden als in der Norm. Bei genauerer Beobachtung wurde bemerkt, dass die Pupillenweite, sobald der Krampfanfall aufzutreten begann, schnell abnahm, und sich in diesem Zustande mit geringen Schwankungen während des Anfalls anhielt, jedoch mit dem Nachlassen der Krämpfe so weit vergrösserte wie vor dem Einsetzen derselben. Dass diese Veränderungen hauptsächlich zentralen Ursprungs sind, wird dadurch erwiesen, dass das ausgeschnittene Froschauge, auch in $0,5 \%$ ige Giftlösung getaucht, keine Pupillenerweiterung zeigt.

\section{Die Hautsekretion.}

Bei der Darreichung dieses Giftes steigert sich die Hautsekretion im Stadium der bulbären und spinalen Krämpfe bedeutend. Aber bei Tieren mit einseitig durchschnittenem $N$. ischiadicus zeigt sich die gesteigerte Hautabsonderung an allen anderen Stellen des Körpers ausser dem Schenkel der operierten Seite, was zeigt, dass die Hautsekretion keine peripher bedingte ist. Auch bei Tieren, bei denen das verlängerte Mark vom Rückenmark abgetrennt ist, wird in der Zeit, wo die bulbären Krämpfe auftreten müssten, die Sekretion gar nicht gefördert; diese erfolgt jedoch mit der Steigerung der spinalen Reflexe. Trotzdem die Sekretionssteigerung immer mit den Krämpfen einhergeht, steht diese mit den Krämpfen nicht in ursächlicher Beziehung, da deise auch bei den curarisierten Tiere erfolgt. Man muss daher annehmen, dass die Sekretionsnervenzentren in der Medulla ob- 
longata und im Rückenmarke mit den Krampfzentren zusammen durch das Gift erregt wurden. Aus den oben geschilderten Resultaten zu schliessen, ist sowohl die anfängliche, wie auch die spätere Sekretionssteigerung auf die Erregung des im verlängerten resp. Rückenmark befindlichen Sekretionssnervenzentrums durch dieses Gift zurückzuführen.

\section{Wirkung des Coriatoxins.}

Das Coriatoxin ist in der Wirkung auf den Frosch im Wesen dem Coriariatoxin gleich, aber in der Intensität von demselben verschieden. Die kleinste letale Dose beträgt nämlich bei subkutaner Injektion auf $100 \mathrm{~g}$ Körpergewicht der Rana nigromaculata ca. $4,2 \mathrm{mg}$; seine Giftigkeit entspricht also nur einem Siebentel der des Coriariatoxins. Wie oben bereits erwähnt, verläuft die Vergiftung mit dem letzteren bereits äusserst langsam, mit dem Coriatoxin jedoch noch langsamer. Bei der Darreichung desselben in der kleinsten letalen Dose vergehen bis zum Erscheinen deutlicher Krampfanfälle 2 Stunden, was wohl daher rührt, dass es wegen seiner gegenüber dem Coriariatoxin bei weitern geringeren Wasserlöslichkeit, schwerer zur Resorption gelangt.

\section{Zusammenfassung.}

Die hauptsächlichen Wirkungen des Coriariatoxins und Coriatoxins lassen sich folgendermassen zusammenfassen: In Anfangsstadium erregen sie wie das Pikrotoxin, Coriamyrtin und Tutin die Medulla oblongata unter Erzeugung einer Frequenzzunahme der Atembewegungen, der sog. Pikrotoxinkrämpfe und Steigerung der Hautsekretion. An den Krämpfen beteiligt sich das Rückenmark nicht, wodurch sich die beiden Gifte von dem Pikrotoxin unterscheiden. Auch wirken sie im Gegensatz zum Pikrotoxin nicht stark auf die Herzhemmungsnerven.

Im späteren Stadium d.h. nach dem Verlauf und Schwund der oben geschilderten Symptome steigert sich aufs neue die Erregbarkeit des Rückenmarks und es stellen sich strychninähnliche Krämpfe sowie abermalige Steigerung der Hautsekretion ein.

Im Terminalstadium findet zentrale motorische Lähmung statt und unter Herzstillstand erfolgt der letale Ausgang.

Das Coriariatoxin und Coriatoxin unterscheiden sich aber in der Wirkungsstärke von einander, indem die tödliche Dose des ersteren Siebentel so klein ist, als die des letzteren. 


\section{Literature.}

(1) Miyachi, Gun'idan-Zassi, 1932, 903.

(2) Kariyone u.S ato, Journ. of Pharmaceut. Soc. of Jap., (jap.) 1930, Nr. 576, $106 ; 1930$, Nr. 581, 659 .

(3) Gottlie b, Arch. f. exp. Path. u. Pharm., 1892, 30, 21.

(4) Marshall, Journ. of pharm. and exp. therap., 1912, 4, 135.

(5) Guinard u. Dumarest, Arch. internat. de pharmacodyn. et de ther., 1899, 6,283 u. 403.

(6) Röber, Arch. f. Anat., Physiol. u. wissensch. Med., 1869, 38. 